\title{
Les relations amicales de A. von Humboldt avec Guillaume Dupuytren
}

\author{
Par Jean Théodoridès, Paris
}

Dans son excellente biographie de Dupuytrens, le regretté $D^{r}$ L. DelHOUME $^{1}$ rappelle les épisodes assez mouvementés ayant précédé, comme il est de règle en pareil cas, l'élection du célèbre chirurgien à l'Académie des Sciences de l'Institut de France dans la Section de médecine et chirurgie.

Dupuytren se présenta à deux reprises en 1825 à cette compagnie: une première fois, le 21 février, au fauteuil de Joseph-François-Louis DescHAMps (1740-1824), chirurgien en chef de l'hôpital de la Charité, mais ce fut son vieux maître le Baron Alexis Boyer (1760-1833) qui fut élu².

Une seconde vacance se produisit à la mort du Baron Pierre Françors Percy (1754-1825) décédé le 18 février 1825, et cette fois Dupuytren l'emporta le 4 avril de la même année par 41 voix sur 59 votants.

Au cours de ces deux campagnes, les amis de Dupuytren s'efforcèrent de lui faire obtenir le maximum de voix en intervenant auprès de leurs collègues des diverses sections de l'Académie des Sciences.

Parmi ceux-ci figure le célèbre savant allemand Alexander von HumBOLDT qui séjournait alors à Paris et fréquentait assidûment l'Académie des Sciences dont il était associé étranger depuis 1810.

On connaît peu de choses sur les relations entre Humboldt et Dupuytren qui n'est pas mentionné parmi les savants français amis de Humboldt dans ses biographies les plus complètes ${ }^{3}$.

Dans son ouvrage sur Dupuytren, le $\mathrm{D}^{\mathrm{r}}$ Delhoume a cependant publié 8 lettres ou billets jusque là inédits que lui adressa Humboldt.

Aucun de ces documents n'est daté, mais on sait par leur contexte qu'ils furent écrits en 1825 , lors des deux candidatures de Dupuytren à l'Institut.

1 L.Delhoume, Dupuytren, 1 vol., 494 p., Baillière, Paris 1935.

2 On trouve dans les Procès-Verbaux des séances de l'Academie des Sciences, tome VIII, (1824-1827), Hendaye 1918 (p.186) le détail de cette élection. Sur 57 votants les nombres de voix furent les suivants: au premier tour: Boyer 26, Serres 14, Dupuytren 13, Larrey 2, Lænnec 1, Jules Cloquet 1; au second tour: Boyer 31, Serres 14, Dupuytren 11, Larrey 1. Des relevés autographes de ces votes sont conservés dans les Archives de l'Académie des Sciences.

3 En particulier celle de H.BEcK, Alexander von Humboldt, 2 vols., 303 et 349 p., SteinerVerlag, Wiesbaden 1959 et 1961. 


\section{Dans l'un d'eux, Humboldt écrit: ${ }^{4}$}

«Vous connaissez mon dévouement, la constance de mon attachement et de mon admiration pour vous. Je ne suis pas des amis qui se sont éloignés de vous. Je suis resté pour vous ce que j'étais il y a 25 ans.»

Le passage nous prouve que les deux savants se connaissaient depuis longtemps et que leur amitié remontait probablement à l'année 1798 au cours de laquelle Humboldt séjourna à Paris d'avril à octobre avant son départ pour le sud de la France, puis l'Espagne d'où il s'embarqua avec Bonpland ${ }^{4 \mathrm{~b}}$ pour son mémorable voyage en Amérique latine (1799-1804).

Le second billet publié par Delhoume ${ }^{5}$ est intéressant, car nous en avons trouvé un recoupement dans une lettre au zoologiste Achille VALEnciennes publiée par nos soins.

Humboldt demande à Dupuytren :

«Pouvez-vous me prêter pour quelques jours Lænnec des moyens d'auscultation.»

Et quelque temps après, dans un billet non daté adressé à Valenciennes ${ }^{6}$, Humboldt lui dit:

"Avant d'acheter le Larrey (sic) sur la percussion de la poitrine que Dupuytren me redemande, j'ose Vous nommer encore une fois l'ouvrage, cher ami: c'est peut-être d'après le nom de l'ouvrage que Vous Vous souviendrez si Vous me l'avez demandé.»

Nous avions noté dans notre édition des lettres de Humboldt à Valenciennes que le savant allemand avait dû commettre un lapsus en écrivant LARREY pour LENNEC.

Le billet publié par Delhoume dont nous n'avions pas eu alors connaissance confirme notre hypothèse.

Les autres billets de Humboldt concernent surtout la première candidature (malheureuse) de Dupuytren.

Il y est en effet question de Boyer (présenté en première ligne avec Dubois), d'Augustin Serres (1786-1868) qui ne sera élu à l'Institut qu'en 1828 et de divers autres candidats.

Humboldt alors à Paris se dépensa sans compter pour Dupuytren, faisant intervenir ses amis Arago, Cuvier, Poisson, Vauquelin, Lacépède, Gay-Lussac, Chaptal, E. Geoffroy Saint-Hilaire, etc...

4 Delhoume, op.cit., p.216. ${ }^{5}$ Delhoume, op.cit., p. 216.

${ }^{4 b}$ Il est possible que ce soit par l'intermédiaire de Bonpland qui était médecin et lié avec Dupuytren que Humbold ait connu ce dernier en 1798.

${ }^{6}$ J.THÉodoridès, Une amitié de savants au siècle dernier: Alexander von Humboldt et Achille Valenciennes (Correspondance inédite), Biol.Méd. $N^{o}$ hors-Série, CXXIX p., Paris 1965 ( $c f . \mathrm{p} . \mathrm{XX})$. 
Le Docteur Delhoume nous avait communiqué un huitième billet inédit de Humboldt à Dupuytren en nous autorisant aimablement à le publier (fig. 1). En voici le texte:
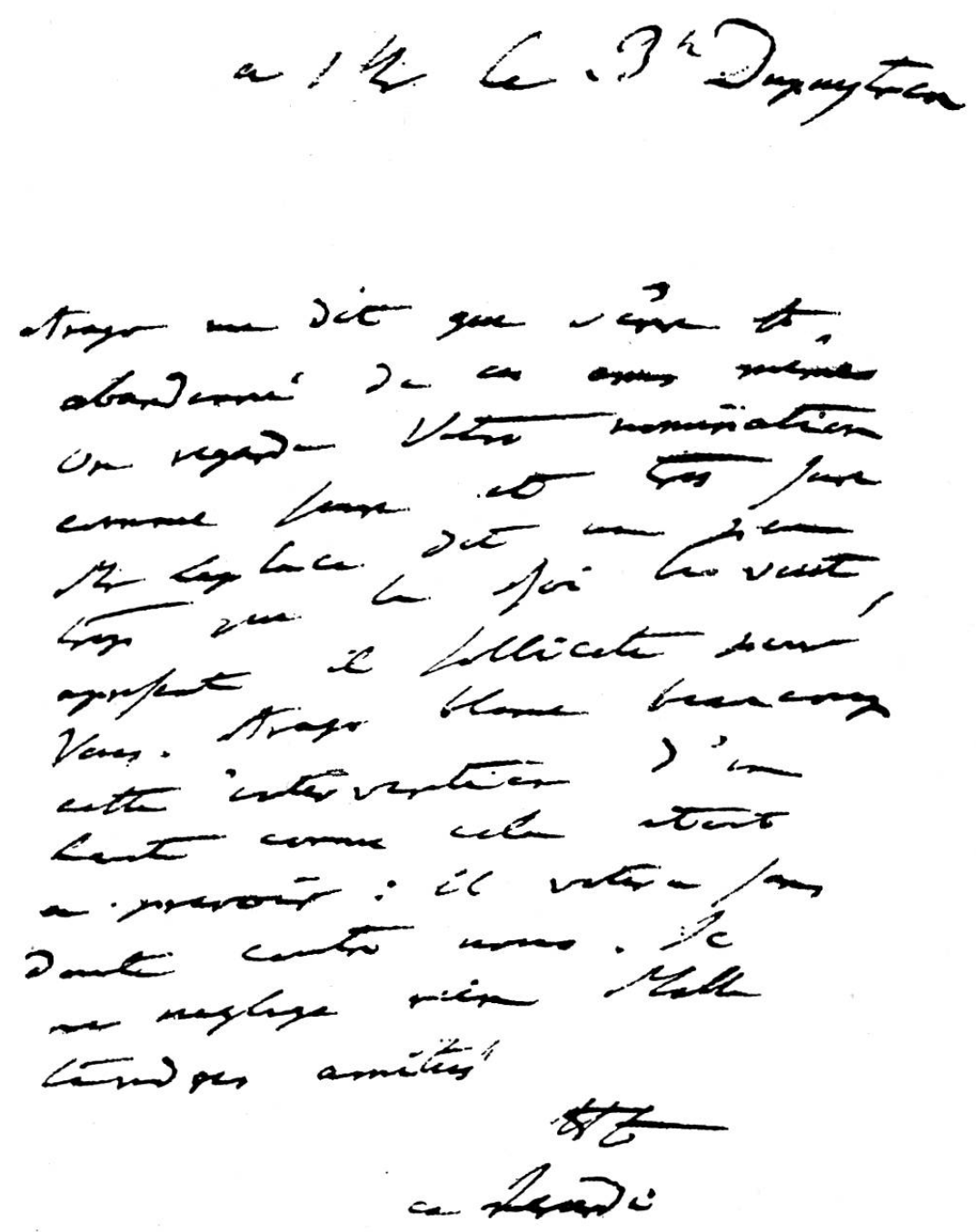

Fig. 1. Billet inédit de Humboldt à Dupuytren

à M. le B[aro]n Dupuytren Arago me dit que Serre (sic) est abandonné de ses amis mêmes. On regarde votre nomination comme sûre et très sûre. M. Laplace dit un peu trop que le Roi la veut, à présent il sollicite pour vous. Arago blâme beaucoup cette intervention d'en haut comme cela était à prévoir: il votera sans doute contre nous. Je ne néglige rien. Mille tendres amitiés.

$\mathrm{H}[$ umbold] $\mathrm{t}$ ce Jeudi

Bien que ces lignes ne soient pas datées, on peut déduire par le contexte qu'elles concernent la seconde candidature de Dupuytren où Serres était encore candidat. 
«L'intervention d'en haut» à laquelle fait allusion Humboldt est celle du roi Charles X dont Dupuytren était le Premier chirurgien. Cette intervention ne laissa pas de trace écrite, et nous remercions très vivement Madame Gauja, archiviste de l'Académie des Sciences, qui a fait pour nous des recherches à ce sujet.

On trouve cependant dans le dossier du Comité Secret, réuni à la suite de la séance de l'Académie des Sciences du 4 avril 1825, la lettre suivante dans laquelle six des candidats au fauteuil de Percy se désistent en raison de cette intervention. Nous remercions MM. les Secrétaires Perpétuels de l'Académie des Sciences de nous avoir autorisé à publier cet intéressant document dont voici le texte:

\section{à Messieurs les Membres de l'Académie Royale des Sciences}

Messieurs,

Dans le dernier comité secret que l'Académie a tenu à l'occasion de la place vacante dans son sein par la mort de M. le Baron Percy, le président et plusieurs membres ont fait prévaloir l'opinion que l'Académie devait se diriger dans cette circonstance d'après des considérations supérieures tout à fait étrangères aux titres scientifiques du concurrent.

Nos titres scientifiques étant les seuls qui nous ont mérité l'honneur de la présentation et que nous ayions à faire valoir nous croyons devoir nous retirer de ce concours en priant l'Académie d'agréer l'hommage de notre profond respect.

\section{Roux Richerand Larrey}

Le B[aro]n Desgenettes J.Cloquet

Serres

Malgré ce désistement, plusieurs des signataires de cette lettre obtinrent des suffrages: sur 59 votants, Dupuytren recueillit, dès le premier tour de scrutin, 41 voix, Cloquet 1, Alibert 1, Richerand 1, Larrey 3, Roux 5 ( $c f$. Delhoume, op. cit. p. 223). Il y eut 7 bulletins blancs.

Un autre document relatif aux relations amicales entre Humboldt et Dupuytren nous a été signalé par Mr. F. Lange, secrétaire de la A.von Humboldt-Kommission de Berlin, que nous remercions très vivement ici.

Il s'agit d'un billet de Dupuytren destiné au chirurgien allemand Johann Friedrich Dieffenbach (1794-1847) et transmis à ce dernier par l'intermédiaire de Humboldt. Ce document reproduit dans l'ouvrage de Dorow ${ }^{7}$ (fig. 2) porte une inscription de la main de Humboldt qui permet de le dater du 24 octobre 1831, année que Humboldt passa entièrement à Paris.

7 Wilhelm Donow, Faksimile von Handschriften berühmter Männer und Frauen, No 4 , Berlin $1838\left(c f . \mathrm{N}^{\circ} 18 \mathrm{a}\right)$. 
En voici le texte:

r.

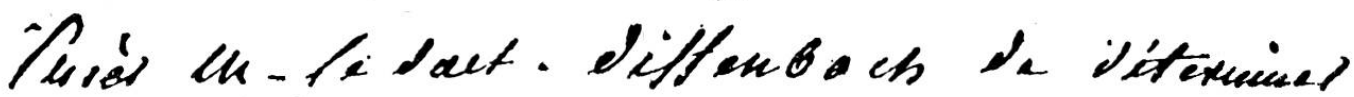

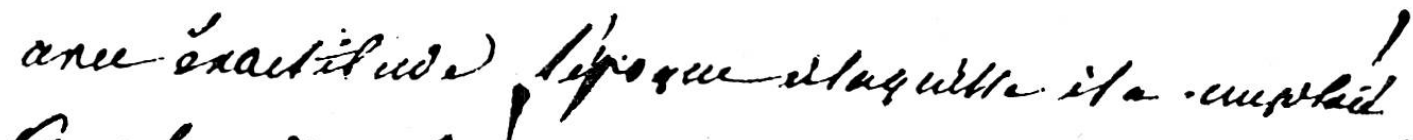

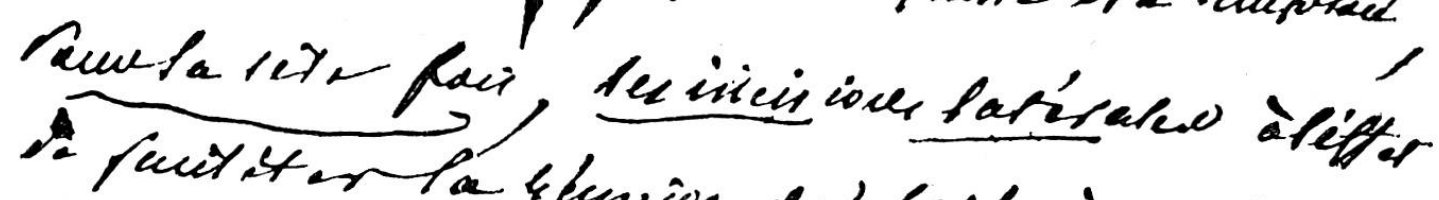

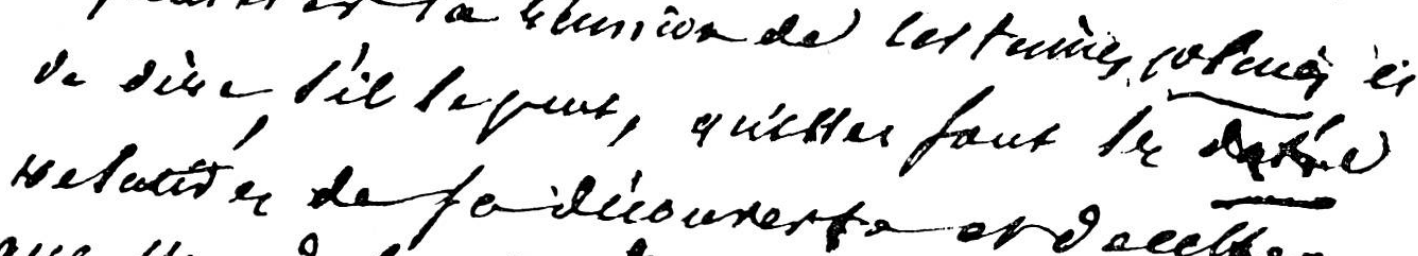

que un-iplect or sector.

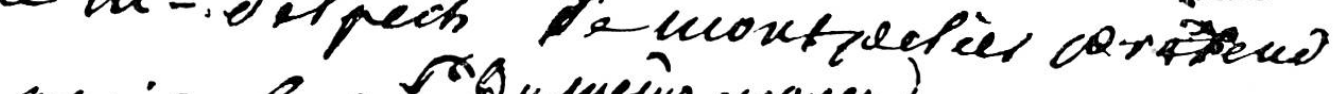

aruis

les question the difsinger

24 oce 189\%

Drough reionte)

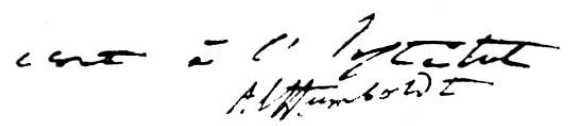

Fig. 2. Billet de Dupuytren à Dieffenbach avec annotations manuscrites de Humboldt

(d'après Donow 1838)

(de la main de Dupuytren):

Priès (sic) M. le doct[eur] Diffenbach (sic) de déterminer, avec exactitude, l'époque à laquelle il a emploié (sic) pour la lère fois, les incisions latérales à l'effet de faciliter la réunion de certaines plaies et de dire, s'il le peut, quelles sont les dates relatives de sa découverte et de celle que M. Delpech ${ }^{8}$ de Montpelier (sic) prétend avoir fait du même moyen il y a cinq ans.

Priès (sic) M.Diffenbach (sic) de faire à ces questions la plus prompte réponse (de la main de Humboldt):

Paris

24. oct[obre] 1831

Dupuytren écrit à l'Institut

Al. Humboldt

8 Jacques-Mathieu Delpech (1777-1832) célèbre chirurgien qui fut Professeur de clinique externe à la Faculté de Médecine de Montpellier. 
Tels sont les documents inédits ou peu connus relatifs aux relations amicales entre Humboldt et Dupuytren que nous voulions présenter ici en hommage au Professeur Ackerknecht à l'occasion de son soixantième anniversaire.

Ils montrent que Humboldt, savant universel, comptait parmi ses amis français non seulement des physiciens et des naturalistes, mais également des représentants - et non des moindres dans le cas de Dupuytren - du monde médical parisien de la première moitié du $\mathrm{XIX}^{\mathrm{e}}$ siècle.

Une étude d'ensemble sur les relations entre Humboldt et les médecins français de son temps reste à écrire. 